Med Clin North Am. 2017 July ; 101(4): 743-753. doi:10.1016/j.mcna.2017.03.006.

\title{
Cervical Cancer Screening
}

\author{
George F. Sawaya, MDa,b, ${ }^{*}$ and Megan J. Huchko, MD, MPH ${ }^{c}$ \\ aDepartment of Obstetrics, Gynecology and Reproductive Sciences, University of California, San \\ Francisco, 550 16th Street, Floor 7, San Francisco, CA 94143, USA \\ ${ }^{b}$ Department of Epidemiology \& Biostatistics, University of California, San Francisco, 550 16th \\ Street, Floor 7, San Francisco, CA 94143, USA \\ 'Department of Obstetrics and Gynecology, Global Health Institute, Duke University, 310 Trent \\ Drive, Box 90519, Durham, NC, 27708, USA
}

\section{Keywords}

Cervical cancer screening; Cervical cancer prevention; Human papillomavirus vaccination; Human papillomavirus testing; Cervical cytology; High-value care; Preterm birth

\section{INTRODUCTION}

Cervical cancer is an uncommon disease in the United States, with an estimated 13,000 incident cases and 4100 deaths occurring in $2016 .{ }^{1}$ Rates have steadily declined over the last few decades coincident with widespread, population-based screening. Disparities in incidence and mortality are still noted, with black and Hispanic women continuing to have higher rates of cervical cancer than white women.

High-quality evidence implicates high-risk human papillomavirus (HPV) types as the causative agents in cervical cancer. HPV infections are common; the US Centers for Disease Control and Prevention (CDC) estimates that nearly all sexually active women are exposed to HPV over their lifetimes. ${ }^{2}$ Although most infections resolve without consequence, persistent infections can lead to precancerous cervical lesions and, in a minority of women, invasive cancer.

The most common precancerous lesions are of squamous cell origin, called cervical intraepithelial neoplasia (CIN), and are graded by the proportion of abnormal epithelium.

- $\quad$ CIN grade 1 indicates an active HPV infection and these lesions are considered low grade with a high spontaneous regression rate; these lesions are generally not treated.

- $\quad$ CIN grade 2 is often considered a high-grade lesion but has a spontaneous regression rate of up to $40 \%$.

*Corresponding author. Department of Obstetrics, Gynecology and Reproductive Sciences, University of California, San Francisco, 550 16th Street, Floor 7, San Francisco, CA 94143., george.sawaya@ ucsf.edu. 
- $\quad$ CIN grade 3 lesions have the highest likelihood of progression to invasion and are universally treated.

The estimated time for CIN grade 3 progression to cancer is on average 10 years, allowing many opportunities for these lesions to be found and treated. Preinvasive lesions of glandular cell origin (adenocarcinoma in situ) are less common but are of such concern that hysterectomy is recommended when diagnosed. Of note, cytologybased screening has led to declines in the incidence and mortality of squamous cell cancer but not in cancers of glandular origin.

High-grade CIN lesions (CIN2 and CIN3) are treated with either ablation (eg, laser, cryotherapy) or excision (eg, loop excision, cone biopsy). ${ }^{3}$ Both treatments have high efficacy (short-term cure rates of $85 \%-95 \%$ ) but have different side effects. The association between excisional procedures and preterm birth has led to a more cautious use of these techniques. Prior systematic reviews have found no associations between cryotherapy and laser ablation and preterm birth. ${ }^{4}$ More recent reviews have noted increases in the risk of preterm birth as excision depths increase as well as small increases with unspecified ablative treatments. ${ }^{5}$ As with much evidence about harm, the observational nature of current studies limits causal inference; the relationship between cervical treatments and preterm birth may be confounded by a third factor affecting risk of both. Acknowledging these potential harms, treatment guidelines by the American Congress of Obstetricians and Gynecologists (ACOG) suggest a judicious approach when treatment is warranted; for example, the guidelines encourage surveillance of CIN2 rather than treatment, especially in young women. ${ }^{6}$

Three highly effective HPV vaccines have been developed to target up to 9 HPV subtypes, covering either the most common oncogenic types (bivalent vaccine against 16/18), or a combination of these plus the condyloma-causing HPV types 6 and 11 (quadrivalent vaccine, now replaced by a nonavalent vaccine). Targeted to adolescents of both sexes, the vaccines have been shown to decrease the incidence of both HPV and CIN, with rates of up to $100 \%$ efficacy against the vaccine-specific HPV types and related disease in women who have not been previously exposed. ${ }^{7,8} \mathrm{HPV}$ vaccines show little cross-protection against other oncogenic subtypes, and effectiveness decreases when administered to older women and women who have previously been exposed to HPV. ${ }^{9}$ Therefore, although the overall population effectiveness is likely to be lower than the efficacy seen in the clinical trials, widespread vaccine uptake is anticipated to result in a decrease in CIN and cervical cancer in the future and may affect the design of screening and treatment programs. ${ }^{10,11}$

\section{SCREENING PROGRAMS IN THE UNITED STATES}

About $90 \%$ of US women report having had screening within the prior 5 years, which is a testament to the acceptability of speculum examinations for collection of cervical specimens. ${ }^{12}$ With screening, it is estimated that the lifetime risk of being diagnosed with cervical cancer in the United States is $0.6 \%{ }^{1}$ Among women developing cancer, $50 \%$ to $60 \%$ have never been screened or not adequately screened, emphasizing the importance of finding innovative ways to provide access to high-quality, low-cost screening to women not engaged in screening programs. ${ }^{13}$ 
As with many cancers, the benefits of cervical cancer screening are well known: decreasing cancer incidence and mortality and perhaps decreasing morbidity to some degree by finding early-stage cancers that are treated with less morbid therapies than those recommended to women with late-stage cancers. In addition, like most cancers, the harms of screening are difficult to measure and often underestimated. For cervical cancer screening, these include false-positive testing, invasive procedures and treatments, psychological distress, and extended surveillance of unclear end. From a societal perspective, costs of various screening strategies need to be understood to ensure that care meets a standard of being high-value. ${ }^{14}$

Cytology-based screening has been the typical approach for decades and has evidence of high effectiveness, but it has been criticized for having a low sensitivity in the detection of high-grade CIN in a single episode of screening. In addition, some common cytologic interpretations (eg, atypical squamous cells of undetermined significance [ASC-US]) have low specificity and positive predictive value. In addition, cytology results lack objectivity, leading to high interobserver variations. In one large US study, agreement between community reading and expert reviews were only moderate for cytologic interpretations (kappa, 0.46; 95\% confidence interval, 0.44-0.48). ${ }^{15}$

The introduction of reliable, reproducible tests for the detection of 13 or 14 HPV types implicated in cervical cancer (high-risk HPV [hrHPV]) has expanded options for screening. Summary evidence indicates that hrHPV testing has a higher sensitivity than cytology in detecting high-grade $\mathrm{CIN}^{16}$ and can be useful in increasing the specificity of some test interpretations (eg, ASC-US). ${ }^{17}$

\section{CURRENT SCREENING STRATEGIES: AVERAGE-RISK WOMEN}

In 2012, ACOG, ${ }^{18}$ the US Preventive Services Task Force (USPSTF), ${ }^{19}$ and the American Cancer Society (ACS) in collaboration with the American Society of Colposcopy and Cervical Cytology (ASCCP) and the American Society of Clinical Pathologists (ASCP) 20 published similar screening guidelines; ACOG updated its guidelines in $2016^{6}$ (Table 1). The guidelines focus on average-risk women, defined as women with no prior diagnosis of CIN2 or a more severe lesion or cervical cancer (CIN21), women who are not immunocompromised (eg, infected with human immunodeficiency virus [HIV]), and women with no in utero exposure to diethylstilbestrol.

All guidelines agree that screening should not begin before age 21 years, regardless of sexual history, and that it be performed no more often than every 3 years. Decision analyses commissioned by the USPSTF indicated that screening more frequently than every 3 years confers small additional reductions in cancer risk, but incurs substantially more screening harms, including false-positive testing and colposcopies. ${ }^{21}$ To mitigate harms, ACOG and ACS/ASCCP/ASCP guidelines specifically discourage annual screening among average-risk women of any age. For women aged 30 years and older, the addition of hrHPV testing to cytology allows the stratification of women with normal cytology and negative HPV tests into a particularly low-risk group in which the frequency of screening can be extended to 5 years, and for identifying women with mild cytologic changes (eg, ASC-US) whose 
underlying risk of CIN21 is high enough to refer to colposcopy when HPV testing is positive.

All guidelines agree that screening can end at age 65 years of age if the following criteria are met: 3 consecutive negative cytology results or 2 consecutive negative cytology plus hrHPV tests within 10 years before cessation of screening, with the most recent test performed within the last 5 years. The ACS/ASCCP/ASCP guidelines state that once screening has been discontinued, it should not be restarted, regardless of the acquisition of new sexual partners.

\section{CURRENT SCREENING STRATEGIES: HIGHER-RISK WOMEN}

Guidelines exclude women at higher than average risk: immune-compromised women, those with prior high-grade CIN or cancer, and those with in utero exposure to diethylstilbestrol.

The Panel on Opportunistic Infections in HIV-Infected Adults and Adolescents recommends beginning screening women infected with HIV at the onset of sexual activity and no later than age 21 years, and continuing over a lifetime (not ending at 65 years of age). ${ }^{22}$ The panel suggests annual screening with cytology alone or cytology plus hrHPV testing for women aged 30 years and older. Intervals can be lengthened to 3 years among those with 3 prior normal cytology tests or 1 normal cytology test and a negative HPV test result. The 2016 ACOG guidelines support this approach and state that it is reasonable to screen women immune-compromised for non-HIV reasons similarly starting at age 21 years. They recommend that women exposed to diethylstilbestrol in utero be screened annually, with no rationale provided.

\section{CURRENT SCREENING STRATEGIES: LOW-RISK WOMEN}

Women who have had surgical removal of the cervix have no risk of cervical cancer. Thus, current guidelines discourage screening among women after hysterectomy with no prior history of high-grade CIN or cancer. The USPSTF gives this a grade D recommendation (harms outweigh benefits). In women with high-grade CIN, ACOG recommends continued routine screening with cytology every 3 years for 20 years after the initial posttreatment surveillance period. This recommendation is more conservative than their 2003 recommendation suggesting screening cessation after 3 normal annual vaginal cytology tests.

Although it is anticipated that HPV vaccination will reduce the incidence of CIN and cancers, the lack of evidence of effectiveness at the population level has led guideline groups to recommend no change in the screening approach to vaccinated women. A recent decision analysis suggests that delaying screening initiation among vaccinated women and continuing with screening less often than every 3 years would be a cost-effective approach. ${ }^{11}$

\section{CONTROVERSIES}

Cancer screening guidelines are designed to maximize benefits and minimize harms, all at a reasonable cost. Frequent screening, earlier ages to begin, later ages to end, and more sensitive tests all contribute to screening effectiveness but, if untethered, also exacerbate 
screening harms and contribute to low-value care. Although current guidelines largely align, there is no consensus as to whether one screening approach should be preferred to another. ACS/ASCCP/ASCP recommend that cytology plus hrHPV testing (cotesting) should be preferred to cytology alone, although the guidelines' authors acknowledge that the evidence supporting the preferred designation is weak. ACOG agrees, justifying cotesting rather than cytology alone by citing evidence that hrHPV testing improves detection of adenocarcinoma. More simply, the USPSTF recommends this strategy be applied to women who would prefer screening less often that every 3 years.

The lack of head-to-head comparisons of cotesting with cytology alone with follow-up algorithms similar to those used in the United States has led to uncertainty with regard to expected outcomes. One Italian trial of 11,810 women aged 25 to 34 years randomized to conventional cytology or liquid-based cytology plus hrHPV testing yielded important results. ${ }^{23}$ After 1 screening round, $17.3 \%$ of cotested women had either an abnormal cytology result or a positive hrHPV result compared with only $4.0 \%$ of women with cytology alone. However, despite such a large increase in positive testing, cotesting led to no additional cases of CIN3 identified but found substantially more cases of CIN1 and CIN2, lesions that are known to regress. Clearly age is an important factor in screening. Although the precise age before which hrHPV testing leads to more harms than benefits is unknown, all current guidelines discourage adding HPV testing to cytology in women less than age 30 years.

In 2014, the US Food and Drug Administration (FDA) approved a stand-alone hrHPV test for primary screening in women aged 25 years and older; this test detects the presence of 1 or more of 14 high-risk HPV types. In response to concerns about excessive colposcopy rates among those testing positive, the approved algorithm triages to colposcopy only those with evidence of HPV types 16 and 18 as well as those with evidence of the 12 other highrisk types who have abnormal cytology. After adjusting for verification bias, the study on which the guideline is based found that colposcopy of everyone with positive hrHPV tests had a sensitivity of $61 \%$ for CIN21; triage with testing for HPV types 16 and 18 reduced the proportion of women undergoing colposcopy but at the expense of sensitivity (45\%). ${ }^{24}$ It is recommended that women with abnormal tests who do not proceed to colposcopy (eg, those with evidence of the 12 other high-risk types who have concurrent normal cytology) have follow-up in 1 year. ${ }^{25}$

The FDA-approved start age of 25 year for stand-alone hrHPV testing is controversial because it contradicts current recommendations by the USPSTF that discourage HPV testing in women less than age 30 years, reflecting the concern that the high prevalence of HPV infection in this age group will lead to oversurveillance and overtreatment. In the study cited earlier, $21 \%$ of women aged 25 to 29 years had positive HPV tests and were referred to colposcopy or placed in surveillance compared with about $7 \%$ screened with cytology alone. 24 


\section{EMERGING NOVEL SCREENING STRATEGIES}

Other novel strategies incorporate hrHPV testing as a first-line stand-alone test. An ongoing trial in Canada (the FOCAL trial) is now randomizing women into 2 arms: (1) hrHPV testing with reflex cytology for those testing positive (women with abnormal cytology get colposcopy), and (2) cytology with reflex hrHPV testing for those ASCUS (women with ASC-US cytology and positive hrHPV testing or LSIL cytology or worse get colposcopy). After a single round, the primary hrHPV screening arm detected more cases of CIN21 (16.5 out of 1000 vs 10.1 out of 1000) and CIN31 ( 7.5 out of 1000 vs 4.6 out of 1000), but required more women to have colposcopy compared with the control arm (58.9 out of 1000 vs 30.9 out of 1000). ${ }^{26}$ Full trial results will provide important evidence on the reach, effectiveness, and cost-effectiveness of this strategy.

In addition to novel screening strategies, innovative screening techniques for hrHPV and cytology screening may be more acceptable to women, potentially broadening the reach among underscreened women. Specifically, self-collection of hrHPV removes the need for a speculum examination, a clinician, and possibly even a clinic visit. Self-collected hrHPV specimens have similar sensitivity and specificity for high-grade CIN to clinician-collected specimens. ${ }^{27,28}$ Although most of the work in self-collection has been done in low-resource settings, multiple studies have shown self-collection to be highly acceptable to women in North America. ${ }^{24,29-31}$ However, fewer studies have shown a relationship between selfcollected HPV and increased screening rates or follow-up referral visits among underscreened women. ${ }^{24}$ Studies of self-collection of cytology specimens have been limited to small pilots, likely because of the theoretic difficulty in obtaining endocervical cells using a vaginal swab and the increasing use of self-collection for HPV testing.

\section{HIGH-VALUE SCREENING}

Clinical guidelines strive to balance benefits and harms in an attempt to make screening high value, at least from the patient's perspective. Clinician surveys monitoring adherence to cervical cancer screening guidelines have been discouraging. In the past, clinicians have had low guidelines adherence, ${ }^{32-34}$ including beginning screening too early ${ }^{35}$; repeating screening more often than indicated ${ }^{35-38}$; and not ending screening in low-risk women, either at age 65 years ${ }^{32,39,40}$ or after hysterectomy for benign disease. ${ }^{41,42}$ Recent studies show a more optimistic picture, suggesting that the age of screening initiation is increasing, ${ }^{43}$ and screening visits for women aged 65 years and older are decreasing. ${ }^{40}$ These changes could be caused by improved adherence to guidelines, patient acceptance of less screening, or changes in reimbursement for services that are not endorsed by guidelines.

Some aspects of cervical cancer screening have been the subject of the Healthcare Effectiveness Data and Information Set (HEDIS) of the National Committee for Quality Assurance. In 2014, a new performance measure entitled "Non-recommended cervical cancer screening in adolescent females" was proposed to capture unnecessary cervical cancer screening. Adding overscreening as a measure of poor-quality care by clinicians may bolster adherence to current guidelines. 
Other definitions of high-value care consider costs more specifically. One major driver of overall costs is screening periodicity. When cervical cancer screening is conducted annually with either cytology alone or cytology in combination with HPV testing, the costeffectiveness has been shown to exceed $\$ 500,000$ per quality-adjusted life year (QALY) gained. ${ }^{44,45}$ Biennial screening has been associated with incremental cost-effectiveness ratios of $\$ 150,000$ to $\$ 200,000$ per QALY gained, ${ }^{44,46,47}$ in part because of the finding that most lesions detected at the frequent screening intervals are those that would regress if left undiscovered and untreated. In contrast, screening conducted every 3 to 5 years has been shown to be associated with less than $\$ 100,000$ per QALY gained. ${ }^{46,48}$ However, all QALY analyses to date have been limited by a lack of a comprehensive set of utilities capturing women's preferences for health states that follow from various strategies. ${ }^{49}$

\section{EVIDENCE GAPS}

It is useful to consider the 6 domains of health care quality put forth by the Institute of Medicine in 2001 when considering the way forward in cervical cancer screening. Clinicians strive to make care safe, effective, patient centered, timely, efficient, and equitable. As new strategies emerge, it will be useful to hold them to these standards to ensure that they are not just newer but also better. Patient-centeredness is key, and better understanding the patient's experience as she proceeds through the screening process will be valuable. Finding ways to make screening acceptable to hard-to-reach groups will realize the greatest impact of screening on cervical cancer incidence. Appropriate HPV vaccination holds great promise for additional protection, especially among sociodemographic groups that may be at risk of being unengaged in future screening settings.

\section{SUMMARY}

Cervical cancer screening in the United States has accompanied profound decreases in cancer incidence and mortality over the last half century. Maintaining gains in cervical cancer prevention requires a continued vigilant approach. Current screening guidelines issued by major groups are largely consistent and strive to find a reasonable balance between benefits and harms by recommending less than annual screening in most women. Attention to minimizing screening harms is an important aspect of all screening and preventive approaches.

As new screening strategies emerge and are adopted, comparative effectiveness analyses will be needed to outline the patient-centered and economic implications of choosing one rather than another. These analyses will be useful for highlighting high-value screening options to clinicians, health systems, and patients. ${ }^{49}$ Above all, providing women with affordable, easily accessible screening, follow-up of abnormal tests, and timely treatment will result in the greatest impact of screening on cervical cancer incidence and mortality.

\section{Acknowledgments}

Disclosure: Dr G.F. Sawaya is funded by NIH (1R01CA169093) to identify the range of reasonable options for cervical cancer screening from a patient-centered and economic perspective. Dr M. J. Huchko is funded by NIH (5R01CA188248 and U54CA190153) to evaluate implementation strategies for cervical screening programs in lowresource settings. The authors declare no commercial or other financial conflicts of interest. 


\section{REFERENCES}

1. Surveillance, Epidemiology, and End Results (SEER) Program of the National Cancer Institute Available at: http://seer.cancer.gov/statfacts/html/cervix.html. Accessed November 26, 2016.

2. Centers for Disease Control and Prevention. Genital HPV infection fact sheet Available at: http:// www.cdc.gov/std/hpv/stdfact-hpv.htm. Accessed November 27, 2016.

3. Sawaya GF, Smith-McCune K. Cervical cancer screening. Obstet Gynecol 2016; 127(3):459-67. [PubMed: 26855089]

4. Kyrgiou M, Koliopoulos G, Martin-Hirsch P, et al. Obstetric outcomes after conservative treatment for intraepithelial or early invasive cervical lesions: systematic review and meta-analysis. Lancet 2006;367(9509):489-98. [PubMed: 16473126]

5. Kyrgiou M, Athanasiou A, Paraskevaidi M, et al. Adverse obstetric outcomes after local treatment for cervical preinvasive and early invasive disease according to cone depth: systematic review and meta-analysis. BMJ 2016;354:i3633. [PubMed: 27469988]

6. Practice bulletin no. 157: cervical cancer screening and prevention. Obstet Gynecol 2016;127(1):e120. [PubMed: 26695583]

7. Petrosky E, Bocchini JA Jr, Hariri S, et al. Use of 9-valent human papillomavirus (HPV) vaccine: updated HPV vaccination recommendations of the advisory committee on immunization practices. MMWR Morb Mortal Wkly Rep 2015;64(11):300-4. [PubMed: 25811679]

8. Lu B, Kumar A, Castellsague X, et al. Efficacy and safety of prophylactic vaccines against cervical HPV infection and diseases among women: a systematic review \& meta-analysis. BMC Infect Dis 2011;11:13. [PubMed: 21226933]

9. Kjaer SK, Sigurdsson K, Iversen OE, et al. A pooled analysis of continued prophylactic efficacy of quadrivalent human papillomavirus (types 6/11/16/18) vaccine against high-grade cervical and external genital lesions. Cancer Prev Res (Phila) 2009;2(10):868-78. [PubMed: 19789295]

10. Herrero R Human papillomavirus (HPV) vaccines: limited cross-protection against additional HPV types. J Infect Dis 2009;199(7):919-22. [PubMed: 19236278]

11. Kim JJ, Burger EA, Sy S, et al. Optimal cervical cancer screening in women vaccinated against human papillomavirus. J Natl Cancer Inst 2017;109(2):1-9.

12. Benard VB, Thomas CC, King J, et al. Vital signs: cervical cancer incidence, mortality, and screening - United States, 2007-2012. MMWR Morb Mortal Wkly Rep 2014;63(44):1004-9. [PubMed: 25375072]

13. NIH consensus statement online 19964 1-3, 43(1): 1-38. Available at: https://consensus.nih.gov/ 1996/1996CervicalCancer102html.htm. Accessed on December 20, 2016.

14. Sawaya GF, Kulasingam S, Denberg TD, et al. Cervical cancer screening in average-risk women: best practice advice from the Clinical Guidelines Committee of the American College of Physicians. Ann Intern Med 2015;162(12):851-9. [PubMed: 25928075]

15. Stoler MH, Schiffman M, Atypical Squamous Cells of Undetermined Significance Low-grade Squamous Intraepithelial Lesion Triage Study Group. Interobserver reproducibility of cervical cytologic and histologic interpretations: realistic estimates from the ASCUS-LSIL Triage Study. JAMA 2001;285(11):1500-5. [PubMed: 11255427]

16. Vesco KK, Whitlock EP, Eder M, et al. Screening for cervical cancer: a systematic evidence review for the U.S. Preventive Services Task Force Rockville (MD); Available at: https:// www.ncbi.nlm.nih.gov/pubmed/22132428. Accessed May, 2011.

17. Arbyn M, Roelens J, Simoens C, et al. Human papillomavirus testing versus repeat cytology for triage of minor cytological cervical lesions. Cochrane Database Syst Rev 2013;(3):CD008054. [PubMed: 23543559]

18. Committee on Practice Bulletins-Gynecology. ACOG practice bulletin number 131: Screening for cervical cancer. Obstet Gynecol 2012;120(5):1222-38. [PubMed: 23090560]

19. Moyer VA. Screening for cervical cancer: U.S. Preventive Services Task Force recommendation statement. Ann Intern Med 2012;156(12):880-91. W312. [PubMed: 22711081]

20. Saslow D, Solomon D, Lawson HW, et al. American Cancer Society, American Society for Colposcopy and Cervical Pathology, and American Society for Clinical Pathology screening 
guidelines for the prevention and early detection of cervical cancer. CA Cancer J Clin 2012;62(3): 147-72. [PubMed: 22422631]

21. Kulasingam SL, Havrilesky L, Ghebre R, et al. Screening for cervical cancer: a decision analysis for the U.S. Preventive Services Task Force. AHRQ publication no. 11-05157-EF-1 Rockville (MD): Agency for Healthcare Research and Quality; 2011.

22. Panel on Opportunistic Infections in HIV-Infected Adults and Adolescents. Guidelines for the prevention and treatment of opportunistic infections in HIV-infected adults and adolescents: recommendations from the Centers for Disease Control and Prevention, the National Institutes of Health, and the HIV Medicine Association of the Infectious Diseases Society of America Available at: http://aidsinfo.nih.gov/contentfiles/lvguidelines/adult_oi.pdf. Accessed November 29, 2016.

23. Ronco G, Giorgi-Rossi P, Carozzi F, et al. Human papillomavirus testing and liquid-based cytology in primary screening of women younger than 35 years: results at recruitment for a randomised controlled trial. Lancet Oncol 2006;7(7): 547-55. [PubMed: 16814206]

24. Duke P, Godwin M, Ratnam S, et al. Effect of vaginal self-sampling on cervical cancer screening rates: a community-based study in Newfoundland. BMC Womens Health 2015;15:47. [PubMed: 26060041]

25. Huh WK, Ault KA, Chelmow D, et al. Use of primary high-risk human papillomavirus testing for cervical cancer screening: interim clinical guidance. J Low Genit Tract Dis 2015;19:91-6. [PubMed: 25574659]

26. Ogilvie GS, Krajden M, van Niekerk D, et al. HPV for cervical cancer screening (HPV FOCAL): complete round 1 results of a randomized trial comparing HPVbased primary screening to liquidbased cytology for cervical cancer. Int J Cancer 2017;140(2):440-8. [PubMed: 27685757]

27. Chen Q, Du H, Zhang R, et al. Evaluation of novel assays for the detection of human papilloma virus in self-collected samples for cervical cancer screening. Genet Mol Res 2016;15(2).

28. Ogilvie GS, Patrick DM, Schulzer M, et al. Diagnostic accuracy of self collected vaginal specimens for human papillomavirus compared to clinician collected human papillomavirus specimens: a meta-analysis. Sex Transm Infect 2005;81(3): 207-12. [PubMed: 15923286]

29. Crosby RA, Hagensee ME, Vanderpool R, et al. Community-based screening for cervical cancer: a feasibility study of rural Appalachian women. Sex Transm Dis 2015;42(11):607-11. [PubMed: 26462184]

30. Scarinci IC, Litton AG, Garces-Palacio IC, et al. Acceptability and usability of self-collected sampling for HPV testing among African-American women living in the Mississippi Delta. Womens Health Issues 2013;23(2):e123-30. [PubMed: 23410619]

31. De Alba I, Anton-Culver H, Hubbell FA, et al. Self-sampling for human papillomavirus in a community setting: feasibility in Hispanic women. Cancer Epidemiol Biomarkers Prev 2008;17(8):2163-8. [PubMed: 18708409]

32. Saint M, Gildengorin G, Sawaya GF. Current cervical neoplasia screening practices of obstetrician/ gynecologists in the US. Am J Obstet Gynecol 2005; 192(2):414-21. [PubMed: 15695980]

33. Corbelli J, Borrero S, Bonnema R, et al. Differences among primary care physicians' adherence to 2009 ACOG guidelines for cervical cancer screening. J Womens Health (Larchmt) 2013;23:397403. [PubMed: 24380500]

34. Perkins RB, Jorgensen JR, McCoy ME, et al. Adherence to conservative management recommendations for abnormal Pap test results in adolescents. Obstet Gynecol 2012;119(6):115763. [PubMed: 22617580]

35. Roland KB, Soman A, Benard VB, et al. Human papillomavirus and Papanicolaou tests screening interval recommendations in the United States. Am J Obstet Gynecol 2011;205(5)(447):e441-8.

36. Berkowitz Z, Saraiya M, Sawaya GF. Cervical cancer screening intervals, 2006 to 2009: moving beyond annual testing. JAMA Int Med 2013;173(10):922-4.

37. Meissner HI, Tiro JA, Yabroff KR, et al. Too much of a good thing? Physician practices and patient willingness for less frequent pap test screening intervals. Med Care 2010;48(3):249-59. [PubMed: 20182268] 
38. Saraiya M, Berkowitz Z, Yabroff KR, et al. Cervical cancer screening with both human papillomavirus and Papanicolaou testing vs Papanicolaou testing alone: what screening intervals are physicians recommending? Arch Intern Med 2010; 170(11):977-85. [PubMed: 20548011]

39. Bellizzi KM, Breslau ES, Burness A, et al. Prevalence of cancer screening in older, racially diverse adults: still screening after all these years. Arch Intern Med 2011; 171(22):2031-7. [PubMed: 22158573]

40. Kale MS, Bishop TF, Federman AD, et al. Trends in the overuse of ambulatory health care services in the United States. JAMA Int Med 2013;173(2):142-8.

41. Cervical cancer screening among women by hysterectomy status and among women aged 65 years - United States, 2000-2010. MMWR Morb Mortal Wkly Rep 2013;61(51-52):1043-7. [PubMed: 23282862]

42. Sirovich BE, Welch HG. Cervical cancer screening among women without a cervix. JAMA 2004;291(24):2990-3. [PubMed: 15213211]

43. Henderson JT, Saraiya M, Martinez G, et al. Changes to cervical cancer prevention guidelines: effects on screening among U.S. women ages 15-29. Prev Med 2013;56(1):25-9. [PubMed: 23137444]

44. Goldie SJ, Kim JJ, Wright TC. Cost-effectiveness of human papillomavirus DNA testing for cervical cancer screening in women aged 30 years or more. Obstet Gynecol 2004;103(4):619-31. [PubMed: 15051550]

45. Kim JJ, Wright TC, Goldie SJ. Cost-effectiveness of alternative triage strategies for atypical squamous cells of undetermined significance. JAMA 2002;287(18): 2382-90. [PubMed: 11988059]

46. Goldhaber-Fiebert JD, Stout NK, Salomon JA, et al. Cost-effectiveness of cervical cancer screening with human papillomavirus DNA testing and HPV-16,18 vaccination. J Natl Cancer Inst 2008;100(5):308-20. [PubMed: 18314477]

47. Mandelblatt JS, Lawrence WF, Womack SM, et al. Benefits and costs of using HPV testing to screen for cervical cancer. JAMA 2002;287(18):2372-81. [PubMed: 11988058]

48. Kim JJ, Sharma M, Ortendahl J. Optimal interval for routine cytologic screening in the United States. JAMA Int Med 2013;173(3):241-2.

49. Sawaya GF, Kuppermann M. Identifying a "range of reasonable options" for cervical cancer screening. Obstet Gynecol 2015;125(2):308-10. [PubMed: 25569012] 


\section{KEY POINTS}

- $\quad$ Cervical cancer screening in the United States has accompanied profound decreases in cancer incidence and mortality over the last half century.

- Current screening guidelines issued by major groups are largely consistent and strive to find a reasonable balance between benefits and harms by recommending less screening in most women.

- Two strategies are endorsed by major US-based guideline groups: (1) triennial cytology for women aged 21 to 65 years, and (2) triennial cytology for women aged 21 to 29 years followed by cytology plus testing for high-risk human papillomavirus types every 5 years for women aged 30 years and older.

- Maintaining gains in cervical cancer prevention requires a continued vigilant approach that includes access to low-cost, high-quality screening for all women and appropriate human papilloma virus vaccination.

- As new screening strategies emerge and are adopted, comparative effectiveness analyses will be needed to outline the patient-centered and economic implications of choosing one rather than another. 


\section{Table 1}

Current recommendations for cervical cancer screening by the US Preventive Services Task Force (2012), American Cancer Society ${ }^{a}$ (2012), and/or American Congress of Obstetricians and Gynecologists (2016)

\begin{tabular}{|c|c|}
\hline \multicolumn{2}{|l|}{ Average-risk Women $^{b}$} \\
\hline Age to begin & $21 \mathrm{y}$ \\
\hline \multirow[t]{4}{*}{ Method and intervals, by age } & Ages 21-65 y: cytology every 3 y \\
\hline & Or \\
\hline & Ages $21-29$ y: cytology every $3 y$, then \\
\hline & Ages 30-65 y: cytology plus hrHPV testing every 5 y \\
\hline Age to end & $65 y^{c}$ \\
\hline \multicolumn{2}{|l|}{ Higher-risk Women (ACOG 2016) } \\
\hline \multirow[t]{4}{*}{ Infected with HIV } & Age to begin: initiation of sexual activity, but no later than age $21 \mathrm{y}$ \\
\hline & Ages $21-29$ y: cytology every year until 3 normal tests, then every 3 y \\
\hline & $\begin{array}{l}\text { Ages } 30-65 \text { y: cytology every year until } 3 \text { normal tests, then every } 3 \text { y, or: cytology plus } \\
\text { hrHPV testing every } 3 \text { y }\end{array}$ \\
\hline & Age to end: none \\
\hline Immunocompromised for non-HIV reasons & Screening beginning at age $21 \mathrm{y}$, then as for women infected with HIV \\
\hline In utero exposure to diethylstilbestrol & Annual cytology screening \\
\hline \multicolumn{2}{|l|}{ Low-risk Women (ACOG 2016) } \\
\hline After total hysterectomy, no prior CIN2 + & Screening should not be performed \\
\hline
\end{tabular}

Abbreviation: HIV, human immunodeficiency virus.

${ }^{a}$ With the American Society of Colposcopy and Cervical Pathology and the American Society of Clinical Pathologists.

$b$

Recommendations apply to women with no prior diagnosis of CIN2 or a more severe lesion or cervical cancer (CIN21), women who are not immunocompromised (eg, HIV infected) and women with no in utero exposure to diethylstilbestrol.

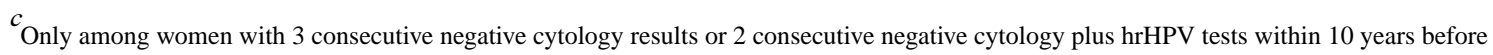
cessation of screening, with the most recent test performed within the last 5 years. 